\title{
Vapor Pressure of Aqueous Solutions of Electrolytes Reproduced with Coarse-Grained Models without Electrostatics
}

\author{
Yamila A. Perez Sirkin, ${ }^{\dagger}$ Matías H. Factorovich, ${ }^{\dagger}$ Valeria Molinero, ${ }^{*},{ }^{\ddagger}$ and Damian A. Scherlis ${ }^{* \dagger}$ \\ ${ }^{\dagger}$ Departamento de Química Inorgánica, Analítica y Quimíca Física/INQUIMAE, Facultad de Ciencias Exactas y Naturales, \\ Universidad de Buenos Aires, Ciudad Universitaria, Pab. II, Buenos Aires C1428EHA, Argentina \\ ${ }^{\ddagger}$ Department of Chemistry, The University of Utah, 315 South 1400 East, Salt Lake City, Utah 84112-0850, United States
}

Supporting Information

ABSTRACT: The vapor pressure of water is a key property in a large class of applications from the design of membranes for fuel cells and separations to the prediction of the mixing state of atmospheric aerosols. Molecular simulations have been used to compute vapor pressures, and a few studies on liquid mixtures and solutions have been reported on the basis of the Gibbs Ensemble Monte Carlo method in combination with atomistic force fields. These simulations are costly, making them impractical for the prediction of the vapor pressure of complex materials. The goal of the present work is twofold:
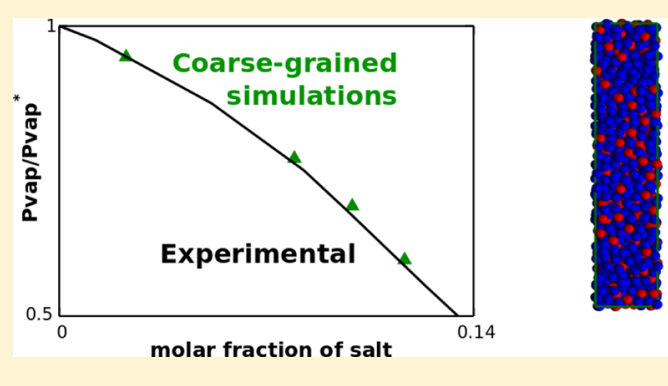

$\mathrm{H}_{2} \mathrm{O}$

Electrolyte

(1) to demonstrate the use of the grand canonical screening approach (Factorovich, M. H. et al. J. Chem. Phys. 2014, 140, 064111) to compute the vapor pressure of solutions and to extend the methodology for the treatment of systems without a liquid-vapor interface and (2) to investigate the ability of computationally efficient high-resolution coarse-grained models based on the $\mathrm{mW}$ monatomic water potential and ions described exclusively with short-range interactions to reproduce the relative vapor pressure of aqueous solutions. We find that coarse-grained models of $\mathrm{LiCl}$ and $\mathrm{NaCl}$ solutions faithfully reproduce the experimental relative pressures up to high salt concentrations, despite the inability of these models to predict cohesive energies of the solutions or the salts. A thermodynamic analysis reveals that the coarse-grained models achieve the experimental activity coefficients of water in solution through a compensation of severely underestimated hydration and vaporization free energies of the salts. Our results suggest that coarse-grained models developed to replicate the hydration structure and the effective ion-ion attraction in solution may lead to this compensation. Moreover, they suggest an avenue for the design of coarse-grained models that accurately reproduce the activity coefficients of solutions.

\section{INTRODUCTION}

Molecular modeling has significantly contributed to understanding equilibrium and transport phenomena in aqueous solutions and polymer electrolyte membranes. The chemical complexity associated with electrolyte solutions in fuel cells, ${ }^{1}$ batteries, biomolecular interfaces, or atmospheric aerosols ${ }^{2,3}$ often makes it difficult to predict their properties. In this context, simulation methods are well suited to provide microscopic insight complementary to the available macroscopic data. Atomistic quantum and classical modeling has been useful to access information on electrode potentials, ion activity coefficients, density profiles, mass transport, mobility, proton exchange, and other properties in solution or in a diversity of electrochemical environments. ${ }^{4-16}$ As for the vapor pressure of aqueous electrolytes, only a few studies have been reported on the basis of the Gibbs Ensemble Monte Carlo method in combination with atomistic potentials. ${ }^{11,16-18}$ Yet, realistic models of modern electrochemical devices typically involve many thousands of atoms that have to be simulated over large time scales. In this scenario, atomistic modeling may turn out to be impracticable, and coarse-grained and multiscale methods start to become indispensable to achieve a complete thermodynamic picture. ${ }^{19-23}$ In recent years, a few coarsegrained models have been developed to calculate the phase diagrams of hydrocarbon mixtures. ${ }^{24,25}$

Various analytical expressions exist to predict the vapor pressure $\left(P_{\mathrm{v}}\right)$ of binary systems. For electrolyte solutions in both diluted and concentrated regimes, the model of Brunauer, Emmett, and Teller (BET) has provided a framework to calculate activity coefficients in bulk, which were successfully applied to predict vapor pressures of aqueous salts. ${ }^{26-28}$ On the other hand, a number of state equations have been derived for mixtures from thermodynamic perturbation theories. ${ }^{29-32}$ Within the realm of molecular simulations, several methodologies have been formulated to determine the point of coexistence and the vapor pressure of liquids. The most widespread of these computational approaches is possibly the Gibbs Ensemble Monte Carlo method by Panagiotopoulos and co-workers on the basis of particle exchange between two reservoirs containing the two phases in equilibrium. ${ }^{33-36}$ Grounded on a different conception, the so-called path-

Received: March 22, 2016

Published: May 19, 2016 
sampling techniques integrate the free energy along a trajectory connecting the two phases of interest. Examples include various schemes such as the expanded ensemble, ${ }^{37}$ simulated tempering, $^{38}$ adaptive umbrella sampling, ${ }^{39}$ and transition matrix Monte Carlo. ${ }^{40}$ Alternately to these somewhat involved techniques, we recently proposed a simple procedure, denoted as grand canonical screening (GCS), to determine the point of liquid-vapor coexistence for systems exposing an interface to the vacuum. ${ }^{41}$ This approach has so far proved useful to study pure bulk phases and aggregates. ${ }^{41,42}$

The purpose of this article is twofold. On the one hand, we illustrate the application of the GCS approach to compute the vapor pressure of solutions. We present an alternative implementation of this methodology that can be applied in the absence of an interface, which can be particularly useful in the case of hydrophobic solutes yielding strongly inhomogeneous profiles in finite liquid slabs. On the other hand, we analyze the free energy contributions related to the vapor pressure of aqueous electrolytes described with coarse-grained models without electrostatic interactions and show how compensation of low hydration and vaporization free energy of the electrolyte leads to the correct equilibrium behavior. The present results provide a general framework for computing liquid-vapor properties of electrolytic systems in physically complex architectures and insights on the development of computationally efficient coarse-grained models that reproduce activity coefficients and the colligative properties that depend on them.

\section{METHODOLOGY}

Coarse-Grained Models. Water was described with coarsegrained model $\mathrm{mW}^{43}$ which represents each molecule as a single particle interacting through anisotropic short-ranged potential favoring "hydrogen-bonded" water structures. This model does not have electrostatic interactions and is based on the short-ranged Stillinger-Weber (SW) potential, ${ }^{44}$ which consists of a sum of two-body attraction terms that favor high coordination and three-body repulsion terms that encourage tetrahedral "hydrogen-bonded" configurations (see Supporting Information). The $\mathrm{mW}$ potential reproduces the energetics, density, and structure of liquid and solid water and its phase transitions with comparable or better accuracy than that of most atomistic models at nearly $1 \%$ of the computational cost. $^{43,45-47}$ In the last few years, this model has proved useful to describe water under a variety of conditions and environments. ${ }^{42,48-61}$

The absolute value of the vapor pressure of $\mathrm{mW}$ water $(0.5$ mbar) is nearly 2 orders of magnitude below the experimental value. ${ }^{41}$ We have shown that this shift, for which the magnitude can be accurately predicted via a statistical thermodynamics analysis, originates in the lack of rotational entropy of the $\mathrm{mW}$ model in the gas phase. ${ }^{41}$ Despite this shift, the liquid-vapor behavior of experimental water is faithfully reproduced by the $\mathrm{mW}$ model in terms of relative vapor pressure $\left(P_{\mathrm{v}} / P_{\mathrm{v}}^{*}\right)$, as it has been assessed for the dependence of equilibrium pressure with respect to temperature, ${ }^{41}$ interface curvature, ${ }^{42}$ or confinement. $^{51}$

We consider here coarse-grained models of salts, $\mathrm{LiCl}$ and $\mathrm{NaCl}$, that have been parameterized with the SW potential to be used with the $\mathrm{mW}$ water force field, without introducing long-range electrostatic interactions. The $\mathrm{LiCl}$ ions were represented by the coarse-grained solute $S$ introduced in ref 52. This species was conceived as a single hydrophilic solute with no charge, which interacts with water via the same potential and parameters as an $\mathrm{mW}$ particle, but setting to zero the angular dependence of the three-body terms centered on the ions. Because this term is repulsive, the net strength of solute-water interactions is larger than that between water molecules. The ion-ion interaction in this model is weak, represented by a pair potential with strength equal to $10 \%$ of that between water and solute. As a result of this choice of parameters, this model quantitatively reproduces the behavior of $\mathrm{LiCl}$ ions in their effect on water structure, melting temperature of ice, and a crossover between crystallization and vitrification as a function of salt concentration. ${ }^{52,62,63}$ The $\mathrm{NaCl}$ ions were modeled using the coarse-grained potential presented in ref 53. This solute yields an accurate representation of the hydration structure and the relative diffusivity of $\mathrm{Na}$ and $\mathrm{Cl}$ ions in solution, and of the experimental association constant of the $\mathrm{Na}^{+}-\mathrm{Cl}^{-}$pair, as well as of the free energy barrier between the solvent-separated and contact ion-pair configurations. ${ }^{53}$ The Yukawa potential was included to mimic the repulsion of ions of the same charge in the case of $\mathrm{NaCl}^{53}$ In both $\mathrm{LiCl}$ and $\mathrm{NaCl}$ models, the interaction between the coarse-grained ions is much weaker than that expected for charged ions. The Supporting Information details the functional form and parameters of the coarse-grained models used in this study.

For consistency with the thermodynamic analysis presented in the last part of the Results and Discussion section, we define the molar fraction of a salt as $x_{\text {salt }}=n_{+} /\left(n_{\mathrm{w}}+n_{-}+n_{+}\right)$and the molar fraction of water as $x_{\mathrm{w}}=n_{\mathrm{w}} /\left(n_{\mathrm{w}}+n_{-}+n_{+}\right)$, where $n_{+}, n_{-}$, and $n_{\mathrm{w}}$ are the number of cations, anions, and water molecules, respectively.

Molecular Simulations. All simulations were carried out using a version of the LAMMPS code ${ }^{64}$ properly modified by us to perform grand canonical molecular dynamics (GCMD) with monoatomic potentials. ${ }^{41}$ GCMD allows for the exchange of water particles between the system and a reservoir to produce the temporal evolution at a controlled chemical potential, $\mu$. The GC/MD ratio was set equal to 20 . The equations of motion were integrated using the Verlet algorithm with a time step of 5 fs. The temperature was controlled with the NoséHoover thermostat at $298 \mathrm{~K}$ with a relaxation time of 0.25 ps. When indicated, the pressure was controlled with a NoséHoover barostat. Periodic boundary conditions were used in all directions.

\section{RESULTS AND DISCUSSION}

Calculation of the Vapor Pressure of Solutions through the GCS Approach. The GCS approach ${ }^{41,42}$ is a simple procedure for the computation of the vapor pressure of finite and bulk systems, with a precision comparable to or better than the one offered by the Gibbs Ensemble Monte Carlo method. To compute the vapor pressure of a pure substance with the GCS approach, various grand canonical simulations must be conducted in the presence of a liquidvapor interface, each one at a different chemical potential around the presumed equilibrium value. If the chemical potential of water $\mu$ fixed in the simulation is above $\mu_{\mathrm{eq}}$, then the number of molecules increases until the simulation box fills completely. Conversely, if $\mu$ is below $\mu_{\mathrm{eq}}$ at the beginning of the simulation, then the number of molecules decreases until all particles have disappeared. By repeating this computational experiment for a given system at different chemical potentials, an upper and a lower bound can be established for $\mu_{\text {eq }}$, which is 
directly linked to $P_{\mathrm{v}}$. We have shown that this methodology can be equally extended to clusters and curved interfaces. ${ }^{41,42}$

There is a fundamental difference in the application of the GCS approach to a solution in comparison to a pure phase. If the initial chemical potential $\mu$ of the solvent is above the one corresponding to equilibrium, then condensation of the solvent will take place. In a solution, this will produce a progressive dilution of the electrolyte, which shall bring with it an increase in $\mu_{\mathrm{eq}}$. Thus, condensation will proceed until $\mu_{\mathrm{eq}}$ equalizes the chemical potential $\mu$ fixed in the simulation, at which point the system will reach equilibrium and thereafter the number of solvent molecules will remain constant. Analogously, if $\mu$ is initially below $\mu_{\text {eq }}$, then the system will evolve via solvent vaporization until the value of $\mu_{\text {eq }}$ drops to the fixed chemical potential. This means that at variance with the case of pure phases the screening of chemical potentials is not needed to locate the point of coexistence of a solution because the system spontaneously evolves to the equilibrium composition for each chemical potential, provided that the cell is large enough to accommodate a liquid-vapor interface.

This behavior is illustrated in Figure 1 for a lithium chloride solution. As mentioned above, in the framework of the GCS

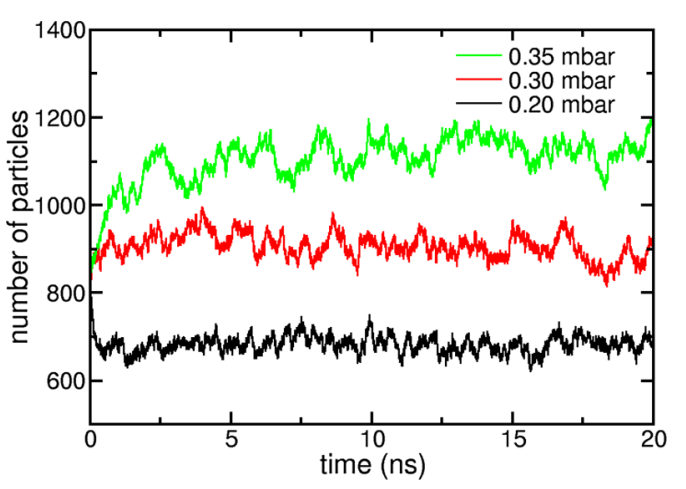

Figure 1. Number of water molecules versus time obtained from GCMD simulations of a slab model of an aqueous $\mathrm{LiCl}$ solution. Each curve starts from the same initial configuration, comprising $820 \mathrm{~mW}$ water molecules and $204 \mathrm{~S}$ ions, simulated at different chemical potentials that correspond to relative vapor pressures of $0.4(0.20$ mbar), 0.6 (0.30 mbar), and 0.7 (0.35 mbar).

scheme, grand canonical simulations at different values of $\mu$ must be carried out in the presence of a liquid-vapor interface, which for a bulk solution implies the use of a liquid slab in contact with a vacuum region. The first system studied consisted of a slab of 820 water molecules and 204 ions in a cell of dimensions $L_{x}=L_{y}=30 \AA$ and $L_{z}=150 \AA$, where $z$ is the direction normal to the interfaces. The liquid slab has initially a thickness of $30 \AA$ in $z$. Figure 1 shows how a larger chemical potential leads to a higher number of solvent molecules or a lower final concentration. For the range of chemical potentials explored, equilibrium is reached within a few nanoseconds.

The strong interaction of $\mathrm{LiCl}$ with water produces an inhomogeneous distribution of ions in the slab along the direction perpendicular to the interfaces, with the concentration in the surface tending to be smaller than that in the bulk in the outer $\sim 1 \mathrm{~nm}^{63}$ We studied solution slabs of different thicknesses to assess the impact of cell size on the vapor pressure of water computed in the presence of liquid-vapor interfaces. Aside from the model with 204 ions and an initial thickness of $150 \AA$ described above, denoted in Figure 2 as the

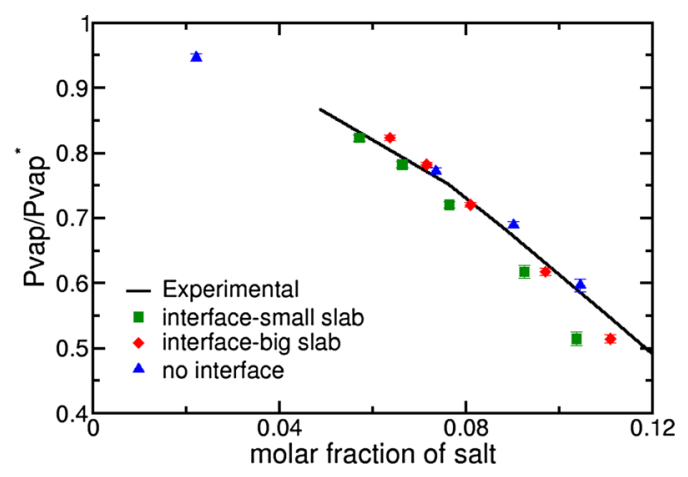

Figure 2. Relative vapor pressure of aqueous $\mathrm{LiCl}$ solutions as a function of the molar fraction of the salt. Experimental data from ref 65 simulation data computed with the GCS method for $\mathrm{mW}$ water and the $\mathrm{S}$ model of $\mathrm{LiCl}^{52}$ Small and big refer to slabs having 820 and 2460 water molecules, respectively, at the beginning of the simulation. The no interface system is equivalent in size to the small slab but without the vacuum region.

small slab, a second liquid slab equal to the first one but 3 times thicker (big slab) was also examined. Figure 2 presents the relative vapor pressure $\left(P_{\mathrm{v}} / P_{\mathrm{v}}^{*}\right)$ obtained for these two models as a function of the molar fraction of the salt. Both exhibit a very similar behavior up to 0.1 molar fraction of salt, falling at the same time very close to the experimental curve. Above this concentration, which is approximately one-half of the experimental solubility of $\mathrm{LiCl}$ at room temperature, the results for the small and large slabs differ, and only the larger slab reproduces quite accurately the experimental data points. As the inhomogeneity induced by water interfaces is always $\sim 1$ $\mathrm{nm},{ }^{66}$ slabs with a thickness of $9 \mathrm{~nm}$ are sufficient to minimize finite-size effects and render accurate vapor pressures in GCS calculations with liquid-vapor interfaces.

Implementation of the GCS Approach without a Liquid-Vapor Interface. Opposite to the case of aqueous $\mathrm{LiCl}$, the local concentration of hydrophobic solutes in water tends to peak near the liquid-gas interface. When dealing with very insoluble substances, such as the hydrophobic polymer electrolytes used for fuel cell membranes, increasing the dimensions of the slab may not be enough to get converged vapor pressures. An approach to the vapor pressure that eliminates the interface altogether would be of help in that scenario. In this section, we propose an alternative implementation of the GCS scheme to compute the equilibrium vapor pressure without the need to include a liquid-vapor interface.

As a first step, the system with no interfaces and with a given composition is equilibrated in the isothermal isobaric NPT ensemble at zero pressure. That run is employed as the starting point for a series of grand canonical simulations at different chemical potentials around the presumed equilibrium value. Along these simulations, however, the mechanical pressure ought to be monitored instead of the number of water particles. If the chemical potential is above $\mu_{\mathrm{eq}}$, then the number of water molecules will tend to increase, which will be reflected in a strong increase of the mean mechanical pressure. The opposite behavior will take place if $\mu$ is smaller than $\mu_{\text {eq }}$, providing that cavitation does not take place. In this way, the average mechanical pressures can reveal the value of the equilibrium chemical potential for a given electrolyte composition. Figure 3 shows how the mechanical pressure is affected by different 
chemical potentials in a model of aqueous $\mathrm{LiCl}$ without interfaces.

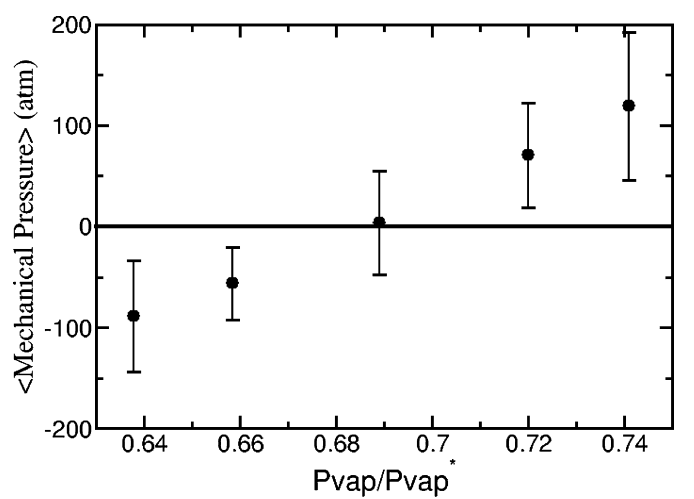

Figure 3. Mean value of the mechanical pressure as a function of the relative vapor pressure for a model of aqueous $\mathrm{LiCl}$ in the absence of interfaces. Each data point has been collected at a different chemical potential. At this composition, the relative pressure corresponding to equilibrium is close to 0.69 .

The vapor pressures of $\mathrm{LiCl}$ solutions obtained through this approach in a simulation cell with 204 ions and initially 820 water molecules are compared in Figure 2 with those calculated from the variation of the number of particles in systems with liquid-vapor interfaces. The method shows excellent accord with the results for the large simulation cell, suggesting that it is a convenient strategy to get rid of boundary effects in the case of solutes presenting strongly inhomogeneous distribution profiles. However, while the elimination of the liquid-vapor interface may be advantageous in some situations, in return this approach reintroduces the need for chemical potential screening to compute every data point on the coexistence curve.

Why Do Coarse-Grained Models Reproduce the Experimental Relative Vapor Pressure? Figure 4 depicts the vapor pressures and associated water activity coefficients calculated without interfaces for $\mathrm{LiCl}$ and $\mathrm{NaCl}$ solutions described with the coarse-grained models, compared to those for an ideal electrolyte, and with the experimental data for the two salts in water. Not only are relative deviations from ideality captured by both models but also these coarse-grained descriptions without electrostatics or other long-range interactions reproduce almost quantitatively the decrease in relative vapor pressure of water in aqueous solutions of increasing concentration. The quality of the description for $\mathrm{NaCl}$ deteriorates for high molar fractions (e.g., for $x_{\text {salt }} \sim 0.09$ or a concentration of 6 molal, the error in the activity coefficient is slightly below $4 \%$ ), but even so the errors in the relative vapor pressures fall below those obtained with various atomistic potentials. ${ }^{11} \mathrm{We}$ were unable to find in the literature previous results for the vapor pressure of aqueous $\mathrm{LiCl}$ based on molecular simulations. However, for other solutes such as $\mathrm{NaCl}$ or $\mathrm{CaCl}_{2}$, recent Gibbs Ensemble Monte Carlo computations using atomistic models have shown typical deviations between 5 and $15 \%$ with respect to the experimental relative vapor pressures. ${ }^{11,18}$ These errors are comparable to or higher than those yielded by the present coarse-grained models at similar concentrations.

The excellent agreement between the coarse-grained predictions and experiments is not only quite remarkable but also puzzling if one notes that the hydration free energy
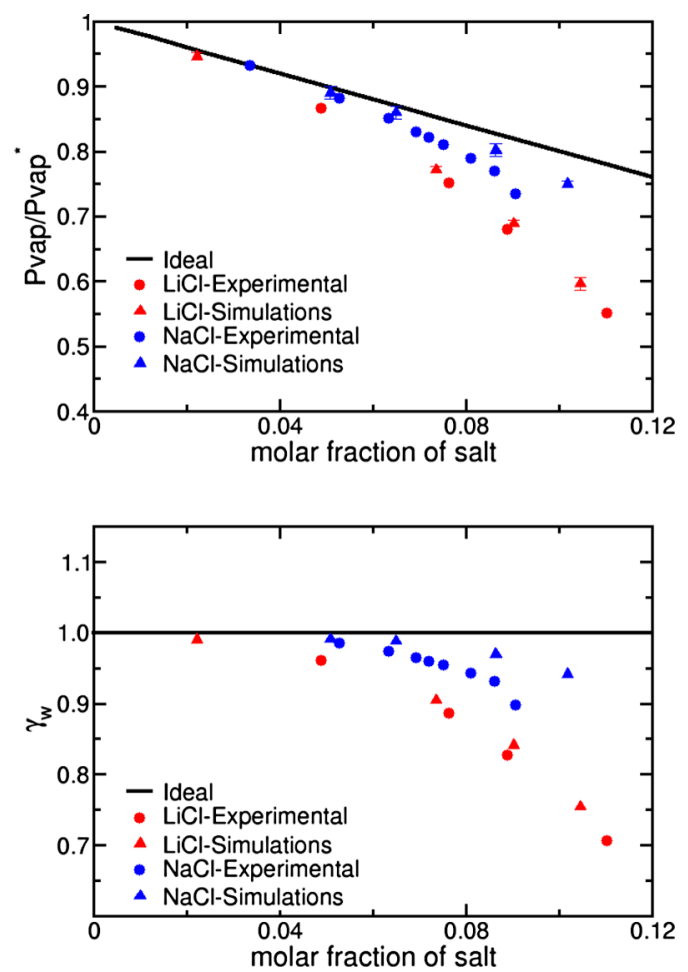

Figure 4. Relative vapor pressure of water (upper panel) and water activity coefficient $\gamma_{\mathrm{w}}$ (lower panel) as a function of the molar fractions of salt for solutions of $\mathrm{LiCl}$ (red) and $\mathrm{NaCl}$ (blue). The triangles show data computed with the GCS method in the absence of liquid-vapor interfaces. The experimental vapor pressures are shown with circles and were taken from refs 65 and 67. The black lines indicate the relative vapor pressures (upper panel) and the activity coefficients (lower panel) for an ideal solution.

( $\Delta G_{\text {hyd }}$ ) of coarse-grained ions is off by an order of magnitude. This quantity can be estimated through the Widom insertion method, whereas the hydration enthalpy $\Delta H_{\text {hyd }}$ can be straightforwardly estimated from the potential energy in molecular dynamics simulations. The thermodynamic properties for the coarse-grained model of lithium chloride are presented in Table 1 together with the experimental values. The model reproduces accurately the relative vapor pressure of water in spite of these serious deviations in the energetics of the solutions. In what follows, we investigate the thermodynamics of the solution with focus on the $\mathrm{LiCl}-$ water mixture to elucidate the origin of this accuracy of the coarse-grained models.

To gain insight on how the relative vapor pressure of the model is right despite the low hydration free energies of the coarse-grained ions, we consider the following thermodynamic cycle, which establishes a connection between the relative vapor pressure and $\Delta G_{\text {hyd }}$ :

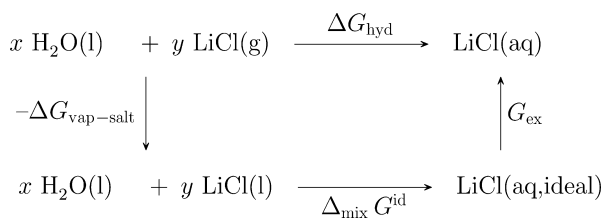

The ideal free energy of mixing $\left(\Delta_{\text {mix }} G^{\text {id }}\right)$ has a purely entropic origin, independent of the parametrization of the model 
Table 1. Thermodynamic Properties of Hydration and Vaporization of $\mathrm{LiCl}(\mathrm{kcal} / \mathrm{mol})$ at Infinite Dilution and $298 \mathrm{~K}^{a}$

\begin{tabular}{lcccccc} 
& $\Delta H_{\text {hyd }}$ & $-T \Delta S_{\text {hyd }}$ & $\Delta G_{\text {hyd }}$ & $\Delta H_{\text {vap-salt }}$ & $-T \Delta S_{\text {vap-salt }}$ & $\Delta G_{\text {vap-salt }}$ \\
\hline experimental & -210.89 & 11.59 & -199.3 & 45.58 & -9.57 & 37.01 \\
coarse-grained model & -22.24 & 7.19 & -15.05 & & &
\end{tabular}

${ }^{a}$ Experimental data from refs 68 and 69.

$$
\Delta_{\text {mix }} G^{\text {id }}=x_{\mathrm{w}} R T \ln x_{\mathrm{w}}+2 x_{\text {salt }} R T \ln x_{\text {salt }}
$$

where the effect of the interactions is accounted for by the excess free energy $G_{\mathrm{ex}}$, which is related to the relative vapor pressure of the electrolyte solution through

$$
\begin{aligned}
G_{\mathrm{ex}} & =x_{\mathrm{w}} R T \ln \gamma_{\mathrm{mW}}+2 x_{\mathrm{salt}} R T \ln \gamma_{ \pm} \\
& =x_{\mathrm{w}} R T \ln \frac{P_{\mathrm{v}}}{x_{\mathrm{w}} P_{\mathrm{v}}^{*}}+2 x_{\mathrm{salt}} R T \ln \gamma_{ \pm}
\end{aligned}
$$

where $\gamma_{\mathrm{mW}}$ is the activity coefficient of the solvent and $\gamma_{ \pm}$is the ionic activity coefficient. These activity coefficients are mutually linked through the Gibbs-Duhem relation ${ }^{70}$

$$
x_{\mathrm{w}} \mathrm{d} \gamma_{\mathrm{w}}+2 x_{\text {salt }} \mathrm{d} \gamma_{ \pm}=0
$$

Thus, because the coarse-grained model provides a correct description of the relative vapor pressure in the range from 0 to $m \approx 8 \mathrm{~mol} \mathrm{~kg}^{-1}$, the excess free energy must be also well captured by the model at least up to that molality.

Given these arguments, we can conclude that the Gibbs energy of mixing, which is the sum of $G_{\text {ex }}$ and $\Delta_{\text {mix }} G^{\text {id }}$, is correctly represented by the coarse-grained model. Now, according to the above thermodynamic cycle, we have

$$
\Delta G_{\text {hyd }}+\Delta G_{\text {vap-salt }}=\Delta_{\text {mix }} G_{\text {ideal }}+G_{\text {ex }}
$$

which means that in the coarse-grained models the overestimation of the hydration free energy must be compensated by an underestimation of a similar magnitude in the vaporization free energy of the salt. More precisely, if $\Delta G_{\text {hyd }}$ for the $\mathrm{LiCl}$ solution is about $185 \mathrm{kcal} / \mathrm{mol}$ less exothermic than the experimental value, then the $\Delta G_{\text {vap-salt }}$ of the model must be close to $-150 \mathrm{kcal} / \mathrm{mol}$ (see Table 1). This implies that the vaporization of liquid $\mathrm{LiCl}$ has to be spontaneous at $298 \mathrm{~K}$, the stable phase of pure $\mathrm{LiCl}$ resulting a gas. We in fact corroborated this prediction through NPT simulations of pure $\mathrm{LiCl}$ at $1 \mathrm{~atm}$ and $298 \mathrm{~K}$, which spontaneously evolved from a condensed phase to a gas phase within 1 ns of dynamics.

For $\mathrm{NaCl}$, we were not able to obtain a reliable estimate of $\Delta G_{\text {hyd }}$ using the Widom method because of the low insertion rate associated with the chloride ion. Preliminary tests, however, suggest that it is much less exothermic than the experimental hydration free energy, in analogy with the case of $\mathrm{LiCl}$. On the other hand, NPT molecular dynamics simulations at $298 \mathrm{~K}$ and 1 bar starting from the $\mathrm{NaCl}$ crystal also end up in complete vaporization. Thus, this compensation mechanism between $\Delta G_{\text {hyd }}$ and $\Delta G_{\text {vap-salt }}$ appears to be general.

These models have not been parametrized to represent the properties of a pure phase but those of an aqueous electrolyte in the context of $\mathrm{mW}$ water, and there is no particular reason why they should reproduce properties of the ionic crystal phase. Instead, the model of lithium chloride replicates quantitatively the experimental phase behavior of aqueous solutions of $\mathrm{LiCl}^{52,62}$ including the depression of the melting point of ice. The model of sodium chloride, on the other hand, was developed to reproduce the hydration structure of the $\mathrm{Na}$ and $\mathrm{Cl}$ ions in $\mathrm{mW}$ water and was shown to also produce the correct association pathway and relative free energies of contact pairs versus solvent separated pairs as well as the ratio of the diffusivity of these two ions in water. ${ }^{53}$ Very recently, it proved accurate to predict the freezing point of concentrated solutions. $^{71}$ The ability of these coarse-grained models to reproduce the freezing point depression of aqueous solutions constitutes an independent corroboration of their ability to reproduce the activity coefficients of water as shown in Figure 4.

The error compensation observed in every case for $\Delta G_{\text {hyd }}$ and $\Delta G_{\text {vap-salt }}$ may look fortuitous, but it is probably not. Both models of aqueous electrolytes bear the correct values of activity coefficients. Equation 4 shows that $\Delta G_{\text {hyd }}$ and $\Delta G_{\text {vap-salt }}$ must exactly compensate for this to happen. Our findings suggest that in the absence of electrostatic interactions a parametrization strategy based on reproducing some fundamental features of the solution, such as solvation structure and effective association between ions, can result in a faithful representation of the activity coefficients of water and ions in solution and all the properties that depend on them. This is possible through compensation of low cohesive energies of hydration and vaporization of the salt resulting from the use of short-ranged interactions in lieu of long-range electrostatics. The short-range interaction potentials of the $\mathrm{mW}$-ion coarsegrained models should be particularly well suited to reproduce activity coefficients of concentrated solutions, for which shortrange interactions dominate the nonideal interactions. ${ }^{26,72-74}$ We note that the Debye lengths of the solutions of the present study would be comparable to or smaller than the diameter of a water molecule. This insight provides a constraint for the development of coarse-grained models of solutions for applications in which colligative properties such as the depression of the vapor pressure and melting temperature or an increase in the boiling point are of interest. In practice, we expect that these models will grasp those physical features imminent to the solution determined by the solvent activity, such as phase transitions, osmotic pressure, and colligative properties in general. Instead, they cannot be expected to provide reliable estimates for thermodynamic parameters involving the nonaqueous salts, hydration or solubility, or to reproduce the concentration dependence of the activity coefficient of the ions in very dilute solutions.

\section{CONCLUDING REMARKS}

We illustrate in this article the application of the GCS approach to compute the vapor pressure of solutions. At variance with the case of pure phases, given a chemical potential and a number of ions in the system, the grand canonical simulations spontaneously evolve to the equilibrium composition. This circumstance spares the need-inherent in pure phases-of multiple runs at different chemical potentials to situate the equilibrium condition, making this scheme natural and straightforward to explore the liquid-vapor behavior of aqueous solutions. In terms of computational cost, this renders the GCS procedure as efficient as the Gibbs Ensemble Monte Carlo method while this is not necessarily true for pure 
systems, where only the later technique finds the point of coexistence in a single simulation. Moreover, we have proposed a modification of the GCS approach on the basis of the mean mechanical pressure, which lifts the requisite of an interface exposed to vacuum to compute the point of coexistence. This approach can be helpful to deal with highly hydrophilic or hydrophobic solutes presenting inhomogeneous density profiles in finite slabs, but in exchange it reinstates the screening of chemical potentials.

Our simulations indicate that the coarse-grained models of aqueous $\mathrm{LiCl}$ and $\mathrm{NaCl}$ based on short-range interactions and developed to work with the $\mathrm{mW}$ water model predict the relative vapor pressure of water in the solutions in very good agreement with experiments, with an accuracy comparable to or above that yielded by atomistic potentials. The present results suggest that these coarse-grained models quantitatively capture the activity coefficients of water in electrolyte solutions regardless of the representation of thermodynamic functions such as vaporization, hydration, or solubility energies and entropies. This attribute-an exceptional accuracy in the activity coefficients coexisting with an unphysical representation of certain thermodynamic variables involving any constituent in a state of aggregation different from the one considered in the parametrization-will likely become a signature of highresolution coarse-grained modeling based on short-range interactions, as these models are extended to systems of increasing complexity.

\section{ASSOCIATED CONTENT}

\section{S Supporting Information}

The Supporting Information is available free of charge on the ACS Publications website at DOI: 10.1021/acs.jctc.6b00291.

The functional form and parameters of the coarsegrained models used in this study (PDF)

\section{AUTHOR INFORMATION}

\section{Corresponding Authors}

*E-mail: valeria.molinero@utah.edu (V.M.)

*E-mail: damian@qi.fcen.uba.ar (D.A.S.)

\section{Funding}

This study has been supported by a collaborative grant of the Agencia Nacional de Promoción Cientifica y Tecnológica of Argentina (PICT 2012-2292 to V.M. and D.A.S.) and by the U.S. Army Research Laboratory under a Cooperative Agreement (Number W911NF-12-2-0023 to V.M).

\section{Notes}

The authors declare no competing financial interest.

\section{ACKNOWLEDGMENTS}

We are thankful to Professors Ernesto Marceca and Gabriel Gordillo for discussions on the thermodynamics of mixtures. We are also thankful to the Center of High Performance Computing of the University of Utah for an award of computing time and technical support.

\section{REFERENCES}

(1) Jeon, S. W.; Cha, D.; Kim, H. S.; Kim, Y. Analysis of the system efficiency of an intermediate temperature proton exchange membrane fuel cell at elevated temperature and relative humidity conditions. Appl. Energy 2016, 166, 165-173.
(2) Zhang, R.; Khalizov, A.; Wang, L.; Hu, M.; Xu, W. Nucleation and growth of nanoparticles in the atmosphere. Chem. Rev. (Washington, DC, U. S.) 2012, 112, 1957-2011.

(3) Andreae, M. O. The aerosol nucleation puzzle. Science 2013, 339, 911-912.

(4) Weber, A. Z.; Newman, J. Modeling transport in polymerelectrolyte fuel cells. Chem. Rev. (Washington, DC, U. S.) 2004, 104, 4679-4726.

(5) Hammes-Schiffer, S.; Soudackov, A. V. Proton-coupled electron transfer in solution, proteins, and electrochemistry. J. Phys. Chem. B 2008, 112, 14108-14123.

(6) Calle-Vallejo, F.; Koper, M. T. C. First-principles computational electrochemistry: achievements and challenges. Electrochim. Acta 2012, $84,3-11$.

(7) Burt, R.; Birkett, G.; Zhao, X. S. A review of molecular modelling of electric double layer capacitors. Phys. Chem. Chem. Phys. 2014, 16, 6519-6538.

(8) Marenich, A. V.; Ho, J.; Coote, M. L.; Cramer, C. J.; Truhlar, D. G. Computational electrochemistry: prediction of liquid-phase reduction potentials. Phys. Chem. Chem. Phys. 2014, 16, 15068-15106.

(9) Carrasco, J.; Hodgson, A.; Michaelides, A. A molecular perspective of water at metal interfaces. Nat. Mater. 2012, 11, 667674.

(10) Hammes-Schiffer, S. Proton-coupled electron transfer: moving together and charging forward. J. Am. Chem. Soc. 2015, 137, 88608871.

(11) Orozco, G. A.; Moultos, O. A.; Jiang, I. G.; Economou, H.; Panagiotopoulos, A. Z. Molecular simulation of thermodynamic and transport properties for the $\mathrm{H}_{2} \mathrm{O}+\mathrm{NaCl}$ system. J. Chem. Phys. 2014, 141, No. 2334507.

(12) Nouri-Khorasani, A.; Malek, K.; Malek, A.; Mashio, T.; Wilkinson, D. P.; Eikerling, M. H. Molecular modeling of the proton density distribution in a water-filled slab-like nanopore bounded by $\mathrm{Pt}$ oxide and ionomer. Catal. Today 2016, 262, 133-140.

(13) Moucka, F.; Bratko, D.; Luzar, A. Salt and water uptake in nanoconfinement under applied electric field: an open ensemble Monte Carlo study. J. Phys. Chem. C 2015, 119, 20416-20425.

(14) Cao, Z.; Peng, Y.; Voth, G. A. Ion transport through ultrathin electrolyte under applied voltages. J. Phys. Chem. B 2015, 119, 75167521.

(15) Moucka, F.; Bratko, D.; Luzar, A. Electrolyte pore/solution partitioning by expanded grand canonical ensemble Monte Carlo simulation. J. Chem. Phys. 2015, 142, No. 124705.

(16) Jiang, H.; Mester, Z.; Moultos, O. A.; Economou, I. G.; Panagiotopoulos, A. Z. Thermodynamic and transport properties of $\mathrm{H}_{2} \mathrm{O}+\mathrm{NaCl}$ from polarizable force fields. J. Chem. Theory Comput. 2015, 11, 3802-3810.

(17) Strauch, H. J.; Cummings, P. T. Gibbs ensemble simulation of mixed solvent electrolyte solutions. Fluid Phase Equilib. 1993, 86, $147-172$.

(18) Tsai, E. S.; Jiang, H.; Panagiotopoulos, A. Z. Monte Carlo simulations of $\mathrm{H}_{2} \mathrm{O}-\mathrm{CaCl}_{2}$ and $\mathrm{H}_{2} \mathrm{O}-\mathrm{CaCl}_{2}-\mathrm{CO}_{2}$ mixtures. Fluid Phase Equilib. 2016, 407, 262-268.

(19) Persson, R.; Nordholm, S.; Perlovich, G.; Lindfors, L. Monte Carlo studies of drug nucleation 1: formation of crystalline clusters of bicalutamide in water. J. Phys. Chem. B 2011, 115, 3062-3072.

(20) Jorn, R.; Savage, J.; Voth, G. A. Proton conduction in exchange membranes across multiple length scales. Acc. Chem. Res. 2012, 45, 2002-2010.

(21) Franco, A. A. Multiscale modelling and numerical simulation of rechargeable lithium ion batteries: concepts, methods and challenges. RSC Adv. 2013, 3, 13027-13058.

(22) Vishnyakov, A.; Neimark, A. V. Self-assembly in nafion membranes upon hydration: water mobility and adsorption isotherms. J. Phys. Chem. B 2014, 118, 11353-11364.

(23) Jacobson, L. C.; Ren, X.; Molinero, V. Assessing the effects of crowding, pore size, and interactions on electro-osmotic drag coefficients. J. Phys. Chem. C 2014, 118, 2093-2103. 
(24) Mognetti, B. M.; Virnau, P.; Yelash, L.; Paul, W.; Binder, K.; Müller, M.; MacDowell, L. G. Coarse-grained models for fluids and their mixtures: comparison of Monte Carlo studies of their phase behavior with perturbation theory and experiment. J. Chem. Phys. 2009, 130, No. 044101

(25) Lafitte, T.; Avendano, C.; Papaioannou, V.; Galindo, A.; Adjiman, C. S.; Jackson, G.; Müller, E. A. SAFT- $\gamma$ force field for the simulation of molecular fluids: 3 . Coarse-grained models of benzene and hetero-group models of $n$-decylbenzene. Mol. Phys. 2012, 110, $1189-1203$.

(26) Stokes, R.; Robinson, R. Ionic hydration and activity in electrolyte solutions. J. Am. Chem. Soc. 1948, 70, 1870-1878.

(27) Ally, M.; Braunstein, J. BET model for calculating activities of salts and water, molar enthalpies, molar volumes and liquid-solid phase behavior in concentrated electrolyte solutions. Fluid Phase Equilib. 1993, 87, 213-236.

(28) Ally, M. R.; Braunstein, J. Statistical mechanics of multilayer adsorption: electrolyte and water activities in concentrated solutions. $J$. Chem. Thermodyn. 1998, 30, 49-58.

(29) Economou, I. G.; Peters, C. J.; de Swaan Arons, J. Water-salt phase equilibria at elevated temperatures and pressures: model development and mixture predictions. J. Phys. Chem. 1995, 99, $6182-6193$.

(30) Gil-Villegas, A.; Galindo, A.; Whitehead, P. J.; Mills, S. J.; Jackson, G.; Burgess, A. N. Statistical associating fluid theory for chain molecules with attractive potentials of variable range. J. Chem. Phys. 1997, 106, 4168-4186.

(31) Galindo, A.; Gil-Villegas, A; Jackson, G; Burgess, A. N. SAFTVRE: phase behavior of electrolyte solutions with the statistical associating fluid theory for potentials of variable range. J. Phys. Chem. B 1999, 103, 10272-10281.

(32) Held, C.; Sadowski, G. Modeling aqueous electrolyte solutions. Part 2. Weak electrolytes. Fluid Phase Equilib. 2009, 279, 141-148.

(33) Panagiotopoulos, A.; Quirke, N.; Stapleton, M.; Tildesley, D. J. Phase equilibria by simulation in the Gibbs ensemble: alternative derivation, generalization and application to mixture and membrane equilibria. Mol. Phys. 1988, 63, 527-545.

(34) Panagiotopoulos, A. Z. Monte Carlo methods for phase equilibria of fluids. J. Phys.: Condens. Mater. 2000, 12, R25.

(35) Vorholz, J.; Harismiadis, V. I.; Panagiotopoulos, A. Z.; Rumpf, B.; Maurer, G. Molecular simulation of the solubility of carbon dioxide in aqueous solutions of sodium chloride. Fluid Phase Equilib. 2004, $226,237-250$.

(36) Frenkel, D.; Smit, B. Understanding Molecular Simulation, 2nd ed.; Academic Press: San Diego, 2002.

(37) Lyubartsev, A. P.; Martsinovski, A. A.; Shevkunov, S. V.; Vorontsov-Velyaminov, P. New approach to Monte Carlo calculation of the free energy: method of expanded ensembles. J. Chem. Phys. 1992, 96, 1776-1783.

(38) Marinari, E.; Parisi, G. Simulated tempering: a new Monte Carlo scheme. Europhys. Lett. 1992, 19, 451-458.

(39) Mezei, M. Adaptive umbrella sampling: self-consistent determination of the non-Boltzmann bias. J. Comput. Phys. 1987, 68, 237-248.

(40) Swendsen, R. H.; Diggs, B.; Wang, J. S.; Li, S.; Genovese, C.; Kadane, J. B. Transition matrix Monte Carlo. Int. J. Mod. Phys. C 1999, 10, 1563-1569.

(41) Factorovich, M. H.; Molinero, V.; Scherlis, D. A. A simple grand canonical approach to compute the vapor pressure of bulk and finite size systems. J. Chem. Phys. 2014, 140, No. 064111.

(42) Factorovich, M. H.; Molinero, V.; Scherlis, D. A. Vapor pressure of water nanodroplets. J. Am. Chem. Soc. 2014, 136, 4508-4514.

(43) Molinero, V.; Moore, E. B. Water modeled as an intermediate element between carbon and silicon. J. Phys. Chem. B 2009, 113, 4008-4016.

(44) Stillinger, F. H.; Weber, T. A. Computer simulation of local order in condensed phases of silicon. Phys. Rev. B 1985, 31, 52625271.
(45) Jacobson, L. C.; Kirby, R. M.; Molinero, V. How short is too short for the interactions of a water potential? Exploring the parameter space of a coarse-grained water model using uncertainty quantification. J. Phys. Chem. B 2014, 118, 8190-8202.

(46) Lu, J.; Qiu, Y.; Baron, R.; Molinero, V. Coarse-graining of TIP4P/2005, TIP4P-Ew, SPC/E, and TIP3P to monatomic anisotropic water models using relative entropy minimization. J. Chem. Theory Comput. 2014, 10, 4104-4120.

(47) Lupi, L.; Kastelowitz, N.; Molinero, V. Vapor deposition of water on graphitic surfaces: formation of amorphous ice, bilayer ice, ice I, and liquid water. J. Chem. Phys. 2014, 141, 18 C508.

(48) Moore, E. B.; Molinero, V. Structural transformation in supercooled water controls the crystallization rate of ice. Nature 2011, 479, 506-508.

(49) Jacobson, L. C.; Hujo, W.; Molinero, V. Amorphous precursors in the nucleation of clathrate hydrates. J. Am. Chem. Soc. 2010, 132, 11806-11811.

(50) Gonzalez Solveyra, E.; de la Llave, E.; Molinero, V.; Soler-Illia, G. J. A. A.; Scherlis, D. A. Structure, dynamics, and phase behavior of water in $\mathrm{TiO}_{2}$ nanopores. J. Phys. Chem. C 2013, 117, 3330-3342.

(51) Factorovich, M. H.; Gonzalez Solveyra, E.; Molinero, V.; Scherlis, D. A. Sorption isotherms of water in nanopores: relationship between hydropohobicity, adsorption pressure, and hysteresis. J. Phys. Chem. C 2014, 118, 16290-16300.

(52) Le, L.; Molinero, V. Nanophase segregation in supercooled aqueous solutions and their glasses driven by the polyamorphism of water. J. Phys. Chem. A 2011, 115, 5900-5907.

(53) DeMille, R. C.; Molinero, V. Coarse-grained ions without charges: reproducing the solvation structure of $\mathrm{NaCl}$ in water using short-ranged potentials. J. Chem. Phys. 2009, 131, 034107.

(54) Bullock, G.; Molinero, V. Low-density liquid water is the mother of ice: on the relation between mesostructure, thermodynamics and ice crystallization in solutions. Faraday Discuss. 2013, 167, 371-388.

(55) Jacobson, L.; Hujo, W.; Molinero, V. Nucleation pathways of clathrate hydrates: effect of guest size and solubility. J. Phys. Chem. B 2010, 114, 13796-13807.

(56) Jacobson, L. C.; Hujo, W.; Molinero, V. Thermodynamic stability and growth of guest-free clathrate hydrates: a low-density crystal phase of water. J. Phys. Chem. B 2009, 113, 10298-10307.

(57) Johnston, J. C.; Molinero, V. Crystallization, melting, and structure of water nanoparticles at atmospherically relevant temperatures. J. Am. Chem. Soc. 2012, 134, 6650-6659.

(58) Moore, E. B.; Allen, J. T.; Molinero, V. Liquid-ice coexistence below the melting temperature for water confined in hydrophilic and hydrophobic nanopores. J. Phys. Chem. C 2012, 116, 7507-7514.

(59) Qiu, Y.; Molinero, V. Morphology of liquid-liquid phase separated aerosols. J. Am. Chem. Soc. 2015, 137, 10642-10651.

(60) Xu, L.; Molinero, V. Liquid-vapor oscillations of water nanoconfined between hydrophobic disks: thermodynamics and kinetics. J. Phys. Chem. B 2010, 114, 7320-7328.

(61) $\mathrm{Xu}, \mathrm{L}$.; Molinero, V. Is there a liquid-liquid transition in confined water? J. Phys. Chem. B 2011, 115, 14210-14216.

(62) Bullock, G.; Molinero, V. Low-density liquid water is the mother of ice: on the relation between mesostructure, thermodynamics and ice crystallization in solutions. Faraday Discuss. 2013, 167, 371-388.

(63) Hudait, A.; Molinero, V. Ice crystallization in ultrafine watersalt aerosols: nucleation, ice-solution equilibrium, and internal structure. J. Am. Chem. Soc. 2014, 136, 8081-8093.

(64) Plimpton, S. Fast parallel algorithms for short-range molecular dynamics. J. Comput. Phys. 1995, 117, 1-19.

(65) Patil, K. R.; Tripathi, A. D.; Pathak, G.; Katti, S. S. Thermodynamic properties of aqueous electrolyte solutions. 1 . Vapor pressure of aqueous solutions of lithium chloride, lithium bromide, and lithium iodide. J. Chem. Eng. Data 1990, 35, 166-168.

(66) Tobias, D. J.; Stern, A. C.; Baer, M. D.; Levin, Y.; Mundy, C. J. Simulation and theory of ions at atmospherically relevant aqueous liquid-air interfaces. Annu. Rev. Phys. Chem. 2013, 64, 339-359. 
(67) Olynyk, P.; Gordon, A. R. The vapor pressure of aqueous solutions of sodium chloride at 20,25 and $30^{\circ}$ for concentrations from 2 molal to saturation. J. Am. Chem. Soc. 1943, 65, 224-226.

(68) Lamoureux, G.; Roux, B. Absolute hydration free energy scale for alkali and halide ions established from simulations with a polarizable force field. J. Phys. Chem. B 2006, 110, 3308-3322.

(69) NIST. http://webbook.nist.gov (accessed October 8, 2015).

(70) Robinson, R. A.; Stokes, R. H. Electrolyte Solutions, 2nd ed.; Dover: Mineola, NY, 2002; pp 24-39.

(71) Fatemi, S. M.; Foroutan, M. Molecular dynamics simulations of freezing behavior of pure water and $14 \%$ water $-\mathrm{NaCl}$ mixture using the coarse-grained model. Iran J. Chem. Chem. Eng. 2016, 35, 1-10.

(72) Pitzer, K. S. Thermodynamics of electrolytes. I. Theoretical basis and general equations. J. Phys. Chem. 1973, 77, 268-277.

(73) Pitzer, K. S.; Mayorga, G. Thermodynamics of electrolytes. II. Activity and osmotic coefficients for strong electrolytes with one or both ions univalent. J. Phys. Chem. 1973, 77, 2300-2308.

(74) Cohen, M. D.; Flagan, R. C.; Seinfeld, J. H. Studies of concentrated electrolyte solutions using the electrodynamic balance. 1 . Water activities for single-electrolyte solutions. J. Phys. Chem. 1987, 91, 4563-4574. 